BULLETIN (New Series) OF THE

AMERICAN MATHEMATICAL SOCIETY

Volume 43, Number 2, Pages 207-226

S 0273-0979(06)01094-9

Article electronically published on February 15, 2006

\title{
LOCAL ALGEBRAS OF A DIFFERENTIAL QUASIGROUP
}

\author{
MAKS A. AKIVIS AND VLADISLAV V. GOLDBERG
}

\begin{abstract}
The authors provide a summary of results in the theory of differential quasigroups and their local algebras and indicate the relationship of these results to recent work on this subject.
\end{abstract}

\section{INTRODUCTION}

A concept close to the notion of a quasigroup appeared first in combinatorics, when near the end of the eighteenth century (in 1779 and 1782) Euler published two papers [20] and [21] on pairs of mutually orthogonal Latin squares. Euler's quasigroup operation was considered on a discrete set. The notion of quasigroup was introduced by Suschkewitsch [55. The term "quasigroup" was first used by Moufang [34, who considered certain quasigroups with a unit. (Later on such quasigroups were named loops.) The first publication on differentiable (and even analytic) quasigroups is the Mal'cev paper 31.

Differentiable quasigroups are a subject of study in both algebra and differential geometry. In geometry, the theory of differentiable quasigroups is connected with the theory of multidimensional three-webs, and in algebra, it is connected with the theory of Lie groups.

From the latter point of view, quasigroups were first considered in a paper of Mal'cev [31. Sometime later, in [1], Akivis found a canonical decomposition for analytic loops similar to the Campbell-Hausdorff decomposition for Lie groups. In 4], Akivis constructed local algebras of multidimensional three-webs which are also local algebras of differentiable quasigroups associated with those webs. Hofmann and Strambach (see, for example, 25, 26]) named these algebras Akivis algebras.

The differentiable quasigroups differ from the Lie groups only by the fact that multiplication in quasigroups is not associative. As a result, in addition to the operation of commutation, local algebras of differentiable quasigroups have an additional ternary operation, namely the operation of association, which is connected with commutation by the so-called generalized Jacobi identity. This new operation is defined in a third-order neighborhood of a differentiable quasigroup.

It is well-known that every Lie algebra is isomorphic to a subalgebra of commutators of a certain associative algebra. A similar theorem was recently proven by Shestakov (see [50, 51] and [52]). He proved that an arbitrary Akivis algebra can

Received by the editors January 2005 and, in revised form, August 10, 2005.

2000 Mathematics Subject Classification. Primary 20N05; Secondary 53A60, 17D99.

Key words and phrases. Quasigroup, web, local algebra, Akivis algebra.

A preliminary version of this article was presented at the Mile High Conference on Quasigroups, Loops and Nonassociative Systems (University of Denver, Denver, Colorado, July 2005). 
be isomorphically embedded into the algebra of commutators and associators of a certain nonassociative algebra. This solves a problem posed by Akivis [4].

The Lie groups compose a special class of differentiable quasigroups. Other special classes of differentiable quasigroups are Moufang quasigroups, Bol quasigroups and monoassociative quasigroups. In these classes, weakened associativity conditions are satisfied. In local algebras (they are called Mal'cev's algebras, Bol's algebras and monoassociative algebras, respectively) of such quasigroups, some additional identities hold.

A quasigroup $Q$ on a manifold $M=Q \times Q$ determines a three-web $W$ whose foliations are given by the equations $x=a, y=b$ and $x \cdot y=c$, where $a, b, c$ are fixed points on $Q$ and "." denotes the operation of multiplication in the quasigroup $Q$. If the quasigroup $Q$ is an abelian Lie group, then the corresponding three-web $W$ is parallelizable, the Thomsen closure condition $(T)$ holds on $W$ (see Figure 3 ) 1 and all its local algebras are trivial Lie algebras. If the quasigroup $Q$ is a Lie group, then on the corresponding three-web $W$ the Reidemeister closure condition $(R)$ (see Figure 4) holds, and all its local algebras are Lie algebras. If the quasigroup $Q$ is a Moufang quasigroup, then on the corresponding three-web $W$ all three Bol closure conditions hold (see Figures 5 and 6), and all its local algebras are Mal'cev's algebras. If the quasigroup $Q$ is a Bol quasigroup, then on the corresponding threeweb $W$ one of three Bol closure conditions holds, and all its local algebras are Bol's algebras. Finally, if the quasigroup $Q$ is monoassociative, then on the corresponding three-web $W$ the hexagonality closure condition holds, and all its local algebras are monoassociative (see Figure 7).

Three-webs were studied in detail in the work of the participants of the Hamburg mathematical seminar of Blaschke in the 1930s. In most of this work, twodimensional webs were the object of study (see [15]). Three-webs of multidimensional manifolds were studied only in two papers, [16] and [18.

After World War II the investigations initiated in [16] and [18 were developed further by Akivis (see, for example, [12, 8] and [9, where one can find further references). It should be noted that the algebraic theory of quasigroups was intensively developed by Belousov's school in Kishinev (on algebraic theory of quasigroups; see [13] and [17]).

In 4, local algebras of multidimensional three-webs were defined and investigated. These local algebras are also local algebras of differentiable quasigroups connected with three-webs.

In the theory of Lie groups, the third converse Lie theorem is of great importance. This theorem states that a Lie algebra determines a local Lie group in a unique way. In general, for differentiable quasigroups, an Akivis algebra does not determine a local quasigroup uniquely. But the uniqueness takes place for Moufang's and Bol's quasigroups. For them, Mal'cev's and Bol's algebras (which are particular cases of Akivis algebras) determine local Moufang's and Bol's quasigroups in a unique way. As to monoassociative quasigroups, their local Akivis algebras do not determine them uniquely. However, a prolonged Akivis algebra defined in a fourth-order differential neighborhood determines a monoassociative quasigroup uniquely. Note that besides the operations of commutation and association, a prolonged Akivis algebra has two quaternary operations called the quaternators.

\footnotetext{
${ }^{1}$ Here and below in this paragraph we refer to the figures in Section 6 .
} 
In the current paper, we provide a summary of results in the theory of differential quasigroups and their local algebras and indicate the relation of these results to some recent work on this subject (see [50, [51, 52, [53, [54]). In particular, we consider commutators and associators of local loops, local algebras of differentiable quasigroups, prolonged local algebras, the generalization of the third Lie theorem, closed $G$-structures defined by differentiable quasigroups, Shestakov's theorem on linear representations of Akivis' algebras and differentiable quasigroups on Grassmannians.

In this paper we introduce the notions of quasicommutator and quasiassociator of a local loop and consider the prolonged local algebras, which was not done in previous publications on this subject. For the quasigroups related to the classical closure conditions (Thomsen, Reidemeister, Moufang, Bol, hexagonal) for the corresponding three-webs, these algebras define closed $G$-structures. As examples, we consider the quasigroups defined on the Grassmannian $\mathbb{G}(1, n+1)$. We also discuss the generalized third converse Lie theorem for the Moufang, Bol and monoassociative loops.

The theory of differentiable quasigroups has numerous applications to different branches of mathematics and physics. In the current paper we consider only its application to classical algebraic geometry (see Section 8). The application of differentiable quasigroups to the theory of symmetric spaces is considered in the book 29] (see also the recently published book [36]) as well as in the series of papers of Sabinin and Mikheev (see, for example, 42, [43, 44, 45], 46, 44]); see also [41, Ch. 0 and 10). Kikkawa [27] introduced the notion of geodesic loops in a space with an affine connection. Such loops were studied in detail in [39] and [5]. The geodesic loops are connected with the problem of addition of velocities in the theory of relativity. This problem was first posed and considered by Einstein [19. Consequently this problem was considered in numerous works of mathematicians and physicists, in particular, in recently published monographs 23 and 56. Note also that in Chapter 6 of the book [30], the authors discuss applications of Moufang's loops and Mal'cev's algebras in physics.

On the connection of Bol's and Moufang's webs with non-Euclidean geometry, see [6. Note that the loop theory is also connected with the theory of homogeneous spaces. This connection was investigated in a series of papers by Sabinin. The results of these papers are presented in the paper [40].

\section{QUASIGROUPS AND LOOPS}

Let $Q$ be a connected and simply connected differentiable manifold of dimension $r$. Suppose that a binary differentiable operation

$$
z=q(x, y)
$$

which is left invertible and right invertible, is defined on $Q$. Such a manifold $Q(q)$ is said to be a differentiable quasigroup. The inverse operations in $Q(q)$ are denoted by ${ }^{-1} q$ and $q^{-1}$ :

$$
x={ }^{-1} q(z, y), \quad y=q^{-1}(x, z) .
$$

They are also differentiable 2

\footnotetext{
${ }^{2}$ All constructions in this paper are of local nature. A local quasigroup is a differentiable manifold $Q$ on which multiplication is defined not for any two arbitrary points, but if it is defined for two points $a$ and $b$, then it is also defined for two points $x$ and $y$ from sufficiently small
} 
A loop is a quasigroup possessing a unit $e$, i.e., an element $e \in Q$ such that

$$
q(e, y)=y, \quad q(x, e)=x .
$$

A loop differs from a group by the fact that in it the associativity condition

$$
(x \cdot y) \cdot z=x \cdot(y \cdot z)
$$

where $x \cdot y=q(x, y)$, does not necessarily hold.

A quasigroup $Q$ allows us to define a three-web $W$ on the manifold $M=Q \times Q$. Three foliations on $M$ are defined by the equations

$$
x=\text { const. }, \quad y=\text { const., } \quad z=q(x, y)=\text { const. }
$$

If $\operatorname{dim} Q=r$, then $\operatorname{dim} M=2 r$.

Conversely, a three-web $W$ given on a differentiable manifold $M$ of dimension $2 r$ defines on $M$ a binary operation $z=q(x, y)$, which is invertible with respect to the variables $x$ and $y$; i.e., a three-web defines a differentiable quasigroup $Q$.

\section{LOCAL LOOPS}

Let $Q$ be a differentiable quasigroup, let $W$ be a three-web defined by $Q$ on the manifold $M=Q \times Q$, and let $A(a, b)$ be a point on $M$. In a neighborhood of the point $A$, we define the operation

$$
z=u \circ v=q\left[{ }^{-1} q(u, b), q^{-1}(a, v)\right] .
$$

It follows from (1) that the point $e=q(a, b)$ is the unit of the quasigroup $Q(\circ)$ because $u \circ e=u$ and $e \circ v=v$. Thus the operation "o" generates a loop with the unit $e$ on the manifold $Q$. We denote this loop by $l_{A}$ and call it the local loop of the quasigroup $Q$. The loop $l_{A}$ is also a local loop of the three-web $W$ defined on the manifold $M=Q \times Q$ (see [4). In (1) and in what follows, we denote by the "o" the new operation in the quasigroup $Q$ defined in a neighborhood of the point $A$ by formula (1). We shall continue to use the notation "." for multiplication performed not only in local loops $l_{A}$ but also in the entire quasigroup $Q$.

A three-web $W$ on a manifold $M=Q \times Q$ allows us to give a geometric interpretation to the operation of multiplication in the loop $l_{A}$. Namely, let $A \in M$, and let $Q_{u}$ and $Q_{v}$ respectively be two leaves of the first and second foliations of the web $W$ passing through $A$. The leaves of the third foliation of $W$ establish the 1-to-1 correspondence between the leaves $Q_{u}$ and $Q_{v}$ in a neighborhood of the point $A$.

Next, let $u \in Q_{u}$ and $v \in Q_{v}$ be two points on the leaves $Q_{u}$ and $Q_{v}$, and let $F_{u}$ and $F_{v}$ be the leaves of the first and second foliations of $W$ passing through the points $u$ and $v$, respectively (see Figure 1). The leaf $w=$ const. of the third foliation of $W$ passing through the common point $F_{u} \cap F_{v}$ of the leaves $F_{u}$ and $F_{v}$ defines a point $w$ on the leaf $Q_{u}$, and this point $w$ corresponds to the product $u \circ v$ in the loop $l_{A}: w=u \circ v$.

neighborhoods $U_{a}$ and $U_{b}$ of the points $a$ and $b$. Moreover, the point $z=q(x, y) \in U_{c}$, where $c=a \cdot b$. The inverse operations $q^{-1}$ and ${ }^{-1} q$ possess the same properties. An example of a local quasigroup is the quasigroup defined by a triple of hypersurfaces in the projective space $\mathbb{P}^{r+1}$ (see Section 8). 


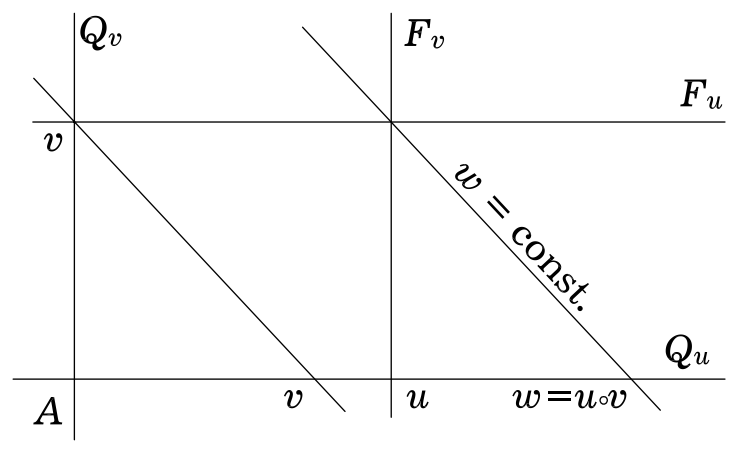

FiguRE 1.

In a neighborhood of a point $A(a, b) \in M$, we define differentiable coordinates $u^{i}, v^{j}, i, j=1, \ldots, r$, such that at point $A$ we have $u^{i}=0, v^{j}=0$. Then we have

$$
\begin{gathered}
z^{i}=l^{i}\left(u^{j}, v^{k}\right), \quad i, j, k=1, \ldots, r, \\
l^{i}\left(0, v^{k}\right)=v^{k}, \quad l^{i}\left(u^{j}, 0\right)=u^{j},
\end{gathered}
$$

where the $l^{i}$ are functions defining the operation "o" in the loop $l_{A}$.

We decompose the right-hand side of equations (2) by means of Taylor's formula. Then by (3) we find that

$$
z^{i}=u^{i}+v^{i}+p_{j k}^{i} u^{j} v^{k}+\frac{1}{2}\left(q_{j k l}^{i} u^{j} u^{k} v^{l}+r_{j k l}^{i} u^{j} v^{k} v^{l}\right)+o\left(\rho^{3}\right),
$$

where $\rho=\max \left(\left|u^{i}\right|,\left|v^{j}\right|\right)$ and $q_{j k l}^{i}=q_{k j l}^{i}, r_{j k l}^{i}=r_{j l k}^{i}$.

\section{Commutators And associators of local LOOPS}

Next, we consider the expressions

$$
\begin{aligned}
& a=u \circ v-v \circ u, \\
& b=(u \circ v) \circ w-u \circ(v \circ w),
\end{aligned}
$$

where $u, v, w \in l_{A}$. These expressions themselves do not belong to the loop $l_{A}$ because they are defined by means of the operation of subtraction, which is not defined in a loop. We shall say that these expressions define the quasicommutator and the quasiassociator of the loop $l_{A}$, respectively.

The coordinates of the quantities $a$ and $b$ are expressed in terms of the coefficients of the decomposition (4) as follows:

$$
\begin{aligned}
& a^{i}=a_{j k}^{i} u^{j} v^{k}, \\
& b^{i}=b_{j k l}^{i} u^{j} v^{k} w^{l},
\end{aligned}
$$

where

$$
\begin{aligned}
& a_{j k}^{i}=p_{j k}^{i}-p_{k j}^{i}, \\
& b_{j k l}^{i}=q_{j k l}^{i}-r_{j k l}^{i}+p_{j k}^{m} p_{m l}^{i}-p_{j m}^{i} p_{k l}^{m} .
\end{aligned}
$$

In a neighborhood of the point $A \in M$, the coordinates $z^{i}, u^{j}, v^{k}, w^{l}$ admit transformations preserving relations (3). Under these transformations, the coefficients of decompositions (4) are not transformed according to the tensor law. However, 
the quantities $a_{j k}^{i}$ and $b_{j k l}^{i}$, which are expressed in terms of the coefficients of (4), are tensors. It follows from (5) that these tensors satisfy the relations

$$
a_{(j k)}^{i}=0, \quad b_{[j k l]}^{i}=2 a_{[j k}^{m} a_{|m| l]}^{i} .
$$

In order to establish the algebraic meaning of the tensors (5), on the loop $l_{A}$ we consider the left and right commutators

$$
a_{l}={ }^{-1}(v \circ u) \cdot(u \circ v), a_{r}=(u \circ v) \circ(v \circ u)^{-1}
$$

and also the left and right associators

$$
b_{l}={ }^{-1}[u \circ(v \circ w)] \circ[(u \circ v) \circ w], \quad b_{r}=[(u \circ v) \cdot w] \circ[u \circ(v \circ w)]^{-1} .
$$

Using (4), one can prove the following theorem:

Theorem 1. The principal parts of the commutators and associators of the loop $l_{A}$ coincide with the quasicommutator a and the quasiassociator $b$ of this loop, i.e., with the expressions

$$
a_{j k}^{i} u^{j} v^{k} \text { and } b_{j k l}^{i} u^{j} v^{k} w^{l}
$$

\section{LOCAL ALGEBRAS OF DIFFERENTIABLE QUASIGROUPS}

We now consider the tangent space $T_{e}$ to the loop $l_{A}$ at its unit $e$. Let $u(t), v(t)$ and $w(t)$ be three smooth curves on the loop $l_{A}$ passing through the point $e$. We assume that $u(0)=v(0)=w(0)=e$. Denote the tangent vectors to these three lines at the point $e$ by $\xi, \eta$ and $\zeta$ (see Figure 2):

$$
\xi=\lim _{t \rightarrow 0} \frac{u(t)}{t}, \quad \eta=\lim _{t \rightarrow 0} \frac{v(t)}{t}, \quad \zeta=\lim _{t \rightarrow 0} \frac{w(t)}{t} .
$$

Suppose that

$$
\begin{aligned}
& \alpha_{l}\left(t^{2}\right)={ }^{-1}(v(t) \circ u(t)) \circ(u(t) \circ v(t)), \\
& \alpha_{r}\left(t^{2}\right)=(u(t) \circ v(t)) \circ(v(t) \circ u(t))^{-1}
\end{aligned}
$$

are the left and right commutators of the curves $u(t)$ and $v(t)$, and

$$
\begin{aligned}
& \beta_{l}\left(t^{3}\right)={ }^{-1}(u(t) \circ(v(t) \circ w(t))) \circ((u(t) \circ v(t)) \circ w(t)), \\
& \beta_{r}\left(t^{3}\right)=((u(t) \circ v(t)) \circ w(t)) \circ(u(t) \circ(v(t) \circ w(t)))^{-1}
\end{aligned}
$$

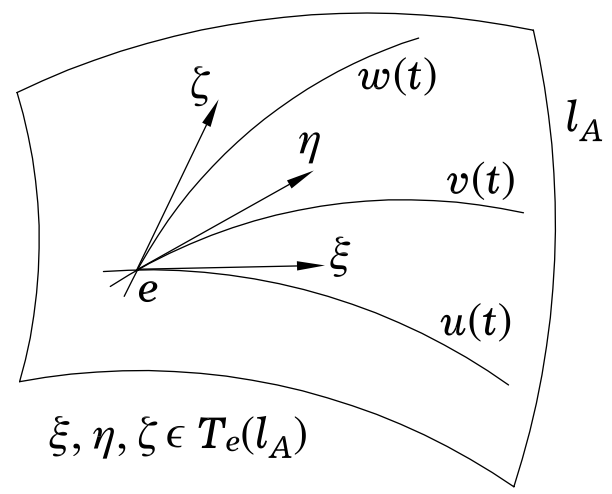

FiguRE 2. 
are the left and right associators of the curves $u(t), v(t)$ and $w(t)$. These four curves also pass through the point $e$.

Theorem 2. The tangent vectors to the curves $\alpha_{l}\left(t^{2}\right)$ and $\alpha_{r}\left(t^{2}\right)$ at the point $e$ coincide, and their coordinates $\alpha^{i}$ are expressed in terms of the coordinates of the vectors $\xi$ and $\eta$ as follows:

$$
\alpha^{i}=a_{j k}^{i} \xi^{j} \eta^{k} .
$$

Similarly, the tangent vectors to the curves $\beta_{l}\left(t^{3}\right)$ and $\beta_{r}\left(t^{3}\right)$ at the point e coincide, and their coordinates $\beta^{i}$ are expressed in terms of the coordinates of the vectors $\xi, \eta$ and $\zeta$ as follows:

$$
\beta^{i}=b_{j k l}^{i} \xi^{j} \eta^{k} \zeta^{l} .
$$

The proof of Theorem 2 is based on the application of Taylor's formula (4) for the decompositions of the product $u \circ v$ as well as of ${ }^{-1} u$ and $u^{-1}$ in a neighborhood of the point $e$.

The vectors $\alpha=\left\{\alpha^{i}\right\}$ and $\beta=\left\{\beta^{i}\right\}$ as well as the vectors $\xi, \eta$ and $\zeta$ belong to the subspace $T_{e}\left(l_{A}\right)$ tangent to the loop $l_{A}$ at its unit $e$ and define in this subspace the operations of commutation and association. These two operations are denoted as follows:

$$
\alpha=[\xi, \eta], \quad \beta=(\xi, \eta, \zeta) .
$$

They satisfy the relations

$$
[\xi, \eta]=-[\eta, \xi]
$$

and

$$
\begin{aligned}
& (\xi, \eta, \zeta)+(\eta, \zeta, \xi)+(\zeta, \xi, \eta)-(\eta, \xi, \zeta)-(\zeta, \eta, \xi)-(\xi, \zeta, \eta) \\
& =[[\xi, \eta], \zeta]+[[\eta, \zeta], \xi]+[[\zeta, \xi], \eta],
\end{aligned}
$$

which follow from (6). The right-hand side of (11) is the Jacobian of the vectors $\xi, \eta$ and $\zeta$. This is the reason that relation (11) is called the generalized Jacobi identity (see 12, Section 2.4 or [8, Section 2.4). The term "generalized" is used here since in the case when a loop $Q$ is a Lie group (i.e., when associativity holds), the identity (11) becomes the Jacobi identity.

Definition 3. The tangent space $T_{e}\left(l_{A}\right)$ in which the operations $[\xi, \eta]$ of commutation and $(\xi, \eta, \zeta)$ of association related by (10) and (11) are defined is called the local algebra of the quasigroup $Q$.

Hofmann and Strambach in 26] considered abstract binary-ternary algebras with operations of commutation and association satisfying relations (10) and (11). Such algebras are called Akivis algebras. Note that the term "Akivis algebra" was introduced by Hofmann and Strambach (see [25] and [26]). They also used the name Akivis identity for the generalized Jacobi identity. Note that in [10] Akivis and Shelekhov used the term "triple system" for the Akivis algebra. In 4], Akivis used the term "local $W$-algebra" for the triple system connected with a three-web. Note also that some particular cases of Akivis algebras were considered by Yamaguti (see [57] and [58]) when he studied symmetric spaces.

Thus, the following theorem is valid.

Theorem 4. The local algebras of a differentiable quasigroup are Akivis algebras. 
This theorem is the generalized direct third Lie theorem for differentiable quasigroups.

However, in general, the third converse Lie theorem is not always true for local differentiable quasigroups, because a local quasigroup is not always uniquely determined by its Akivis algebra (see, for example, [25] or [26], pp. 240-241). In what follows, we shall consider some quasigroups for which the third converse Lie theorem is valid as well as a construction generalizing the third converse Lie theorem.

\section{Prolonged local algebras}

In Taylor's formula (4), considered earlier, we restricted ourselves to terms of at most the third order. However, if a quasigroup is of class $C^{p}, p>3$, or of class $C^{\infty}$ or $C^{\omega}$, then we can prolong formula (4) up to some definite order $p$ or even up to $\infty$. The coefficients of such a prolonged decomposition are the partial derivatives of the function $l(u, v)$ satisfying relations (3). Because now

$$
u=\left(u^{1}, \ldots, u^{n}\right), \quad v=\left(v^{1}, \ldots, v^{n}\right), \quad z=\left(z^{1}, \ldots, z^{n}\right),
$$

condition (3) takes the form

$$
l(0, v)=v, \quad l(u, 0)=u .
$$

As a result, the first three terms of this decomposition coincide with the corresponding terms of decomposition (4), and the remaining terms have the form

$$
z^{i}=u^{i}+v^{i}+p_{j k}^{i} u^{j} v^{k}+\frac{1}{2}\left(q_{j k l}^{i} u^{j} u^{k} v^{l}+r_{j k l}^{i} u^{j} v^{k} v^{l}\right)+\sum_{s=4}^{\infty} P_{s}^{i}\left(u^{j}, v^{k}\right)
$$

where the polynomials $P_{s}^{i}$ are homogeneous of degree $s$ with respect to the variables $u^{j}$ and $v^{k}$. These polynomials can be represented in the form

$$
P_{s}^{i}\left(u^{j}, v^{k}\right)=\sum_{q=1}^{s-1} P_{s, q}^{i}\left(u^{j}, v^{k}\right),
$$

where the polynomials $P_{s, q}^{i}\left(u^{j}, v^{k}\right)$ are of degree $q$ in the variables $u^{j}$ and of degree $s-q$ in the variables $v^{k}$. The above sum does not have the polynomials $P_{s, 0}^{i}$ and $P_{s, s}^{i}$, because by (3) they vanish.

The coefficients of the polynomials $P_{s, q}^{i}\left(u^{j}, v^{k}\right)$ are the partial derivatives of the functions $l^{i}(u, v)$ with respect to the variables $u^{j}$ and $v^{k}$, and this is the reason that these coefficients are not tensors. But by means of the transformation of variables

$$
\widetilde{u}^{i}=\varphi^{i}\left(u^{j}\right), \quad \widetilde{v}^{i}=\psi^{i}\left(v^{j}\right), \quad \widetilde{z}^{i}=\kappa^{i}\left(z^{j}\right),
$$

the polynomials $P_{s, q}^{i}\left(u^{j}, v^{k}\right)$ can be reduced to the form

$$
P_{s, q}^{i}=\frac{1}{s !} \sum_{q=1}^{s-1}\left(\begin{array}{l}
s \\
q
\end{array}\right) a_{j_{1} \ldots j_{q} k_{q+1} \ldots k_{s}}^{i} u^{j_{1}} \ldots u^{j_{q}} v^{k_{q+1}} \ldots, v^{k_{s}},
$$

whose coefficients satisfy the additional condition

$$
a_{\left(j_{1} \ldots j_{q} k_{q+1} \ldots k_{s}\right)}^{i}=0
$$

(see [1]). After this reduction, the coefficients $a_{j_{1} \ldots j_{q} k_{q+1} \ldots k_{s}}^{i}$ become tensors. In the tangent space $T_{e}\left(l_{A}\right)$ to the loop $l_{A}$, each of these tensors defines an $s$-ary operation

$$
\theta_{q}^{i}=a_{j_{1} \ldots j_{q} k_{q+1} \ldots k_{s}}^{i} \xi^{j_{1}} \ldots \xi^{j_{q}} \eta^{k_{q+1}} \ldots \eta^{k_{s}}, q=1, \ldots, s-1,
$$


where $\xi^{j}, \eta^{k}$ and $\theta_{q}^{i}$ are the coordinates of the vectors $\xi, \eta, \theta_{q} \in T_{e}\left(l_{A}\right)$, respectively.

Definition 5. The space $T_{e}\left(l_{A}\right)$, in which besides the operations $[\xi, \eta]$ of commutation and $(\xi, \eta, \zeta)$ of association, the $s$-ary operation (15) is also defined, with $s \geq 4$, is called a prolonged local algebra of the quasigroup $Q$ of order $s$.

We denote such a prolonged local algebra by $L^{(s)}(Q)$. In [47] algebraic structures similar to $L^{(s)}(Q)$ were called hyperalgebras.

In addition to the relations implied by $(14)$, the operations in the algebra $L^{(s)}(Q)$ can satisfy a series of conditions implied by the properties of the quasigroup $Q$ with which this algebra is connected. Characterization of such conditions is rather complex. To date, such a characterization has been carried out only for a few types of quasigroup $Q$. In what follows, we consider some such quasigroups.

In [9] (see also 8]), the authors of the current paper posed the problem of generalizing the Campbell-Hausdorff formula (which is valid for Lie groups and Moufang loops) to Bol loops. G. P. Nagy [35] gave a partial solution to this problem. He constructed the Campbell-Hausdorff formula for local analytic Bruck loops which differ from Bol loops by the fact that for Bruck loops in addition to the Bol identity (see Example 4 in Section 6) the automorphic invertibility property

$$
(x \cdot y)^{-1}=x^{-1} \cdot y^{-1}
$$

holds.

\section{Closed $G$-Structures Defined By differentiable Quasigroups}

Definition 6. A $G$-structure on a smooth manifold $M$ is said to be closed if it is completely defined by a finite number of structure constants.

For example, a Lie group $G^{r}$ is defined by its structure constants $c_{j k}^{i}, c_{k j}^{i}=-c_{j k}^{i}$, satisfying the Jacobi identities. The number of these constants is $n<\frac{1}{2} r^{2}(r-1)$.

Another example is the $G$-structure defined by a homogeneous space $G / H$, where $G$ is the group of motions of the space and $H$ is its stationary subgroup. This $G$ structure is also closed because it depends on a finite number $n$ of constants which can be expressed as the ratio of the number $n_{1}$ of structure constants of $G$ and the number $n_{2}$ of structure constants of $H$.

The notion of a closed $G$-structure was introduced in 1975 in [3].

It is well-known that a Lie algebra $L(G)$ uniquely defines the local Lie group $G$ associated with $L(G)$ - this is the third converse Lie theorem. However, in general, the local algebras $L(Q)$ of a differentiable quasigroup $Q$ do not enjoy this property. A local Akivis algebra or its prolonged algebra uniquely defines a differentiable quasigroup $Q$ only if the $G$-structure related to $Q$ is closed. This may not happen for a local algebra $L(Q)$, but will be true for some prolonged algebra $L^{(s)}(Q)$. In the latter case this prolonged algebra $L^{(s)}(Q)$ uniquely defines a local quasigroup $Q$.

We consider further some special quasigroups and the local algebras associated with them.

1. Commutative Lie groups. For these, we have the conditions

$$
u \circ v=v \circ u, \quad(u \circ v) \circ w=u \circ(v \circ w),
$$

which are satisfied in every local loop $l_{A}$. In this case, formula (4) takes the form $z=u+v$. Thus, the commutators and the associators of local algebras vanish, and the local algebras become trivial. 


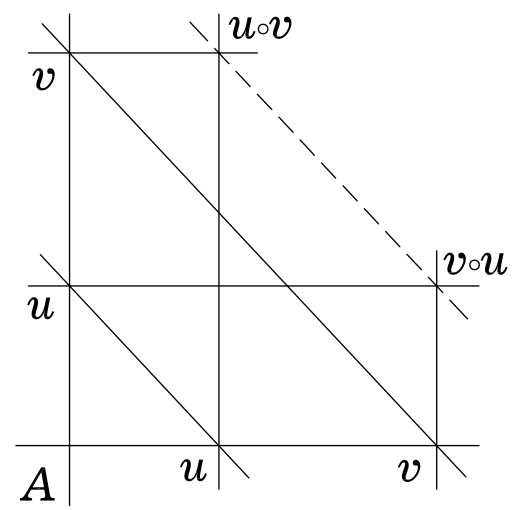

$(T)$

FiguRE 3.

Three-webs defined by such quasigroups are parallelizable, and the Thomsen closure condition $(T)$ (see Figure 3) holds on them (see [15, 12, [8, 24]).

2. Lie groups. For these, in each local loop $l_{A}$, we have

$$
(u \circ v) \circ w=u \circ(v \circ w) \text {. }
$$

Thus, the associators $(\xi, \eta, \zeta)$ of the local algebras vanish. As a result,

$$
J(\xi, \eta, \zeta)=0,
$$

and the local algebras become Lie algebras. Condition (17) shows that the $G$ structure determined by a Lie group is a closed $G$-structure of second order, because the structure constants of a Lie group are completely determined by a second-order neighborhood of its unit. Note that after specialization (14) formula (12) becomes the classical Campbell-Hausdorff formula.

Three-webs defined by Lie groups are called group three-webs, and the Reidemeister closure condition $(R)$ (see Figure 4) holds on them (see [15, 12, 8], 24]).

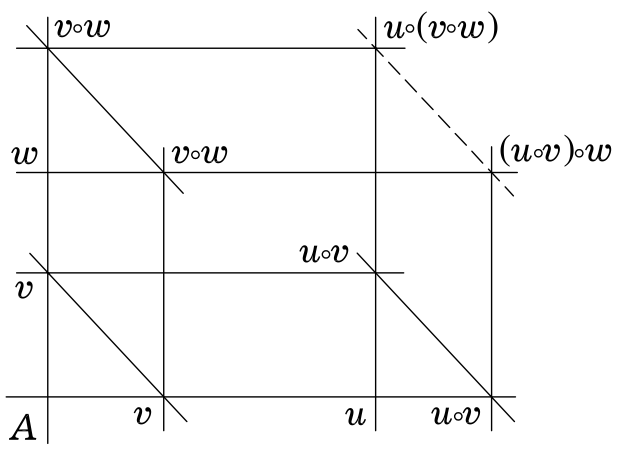

$(R)$

FIGURE 4. 
3. Moufang quasigroups. A Moufang quasigroup is a quasigroup for each local loop of which the condition

$$
(u \cdot v) \cdot(w \cdot u)=u \cdot((v \cdot w) \cdot u)
$$

holds (see [37, p. 89). This relation is called the Moufang identity.

It follows that the associators $(\xi, \eta, \zeta)$ of the local algebras of Moufang quasigroups are skew-symmetric and expressed by means of the commutators as follows:

$$
(\xi, \eta, \zeta)=\frac{1}{6} J(\xi, \eta, \zeta)
$$

Thus, the $G$-structure determined by a Moufang quasigroup is a closed $G$-structure of second order.

Local algebras of Moufang quasigroups have only one independent operation, commutation. Such algebras are called Mal'cev algebras.

As was proven in 48 , this operation of commutation satisfies the identity

$$
[\xi,[\zeta,[\eta, \theta]]]+[\eta,[\theta,[\xi, \zeta]]]+[\zeta,[\eta,[\theta, \xi]]]+[\theta,[\xi,[\zeta, \eta]]]=[[\xi, \eta],[\zeta, \theta]],
$$

which is called the Sagle identity.

If we set $\theta=\xi$ in equation (18), then (18) becomes

$$
[[\xi, \eta],[\xi, \zeta]]=[[[\xi, \eta], \zeta], \xi]+[[[\eta, \zeta], \xi], \xi]+[[[\zeta, \xi], \xi], \eta] .
$$

The last equation characterizes the Mal'cev algebra (see [31] and [28]).

4. Bol quasigroups. A Bol quasigroup is a quasigroup for each local loop of which one of the two conditions

$$
(u \cdot(w \cdot u)) \cdot v=u \cdot(w \cdot(u \cdot v))
$$

or

$$
u \cdot((v \cdot w) \cdot v)=((u \cdot v) \cdot w) \cdot v
$$

holds (see [37, p. 112). The above conditions are called the left Bol identity and the right Bol identity, respectively, and are denoted by $\left(B_{l}\right)$ and $\left(B_{r}\right)$. The corresponding quasigroups are called the left and right Bol quasigroups. For them, the same notations, $\left(B_{l}\right)$ and $\left(B_{r}\right)$, are used.

For $w=e$, the left Bol identity takes the form

$$
(u \circ u) \circ v=u \circ(u \circ v),
$$

and the right Bol identity takes the form

$$
u \circ(v \circ v)=(u \circ v) \circ v .
$$

Note that up to notation, condition (19) is obtained from associativity condition (16) by taking $v=u$ in (16); similarly, condition (20) is obtained from (16) by taking $w=v$ in (16).

Conditions (19) and (20) are the weakened associativity conditions, called the left and right alternativity conditions, respectively.

Besides the left and right $\mathrm{Bol}$ quasigroups, middle Bol quasigroups $\left(B_{m}\right)$ are also considered. They are characterized by the fact that in their local loops the condition

$$
u \circ(v \backslash u)=(u / v) \circ u
$$

holds, where "\" and "/" are the operations in the right inverse loop $l_{A}^{-1}$ and the left inverse loop ${ }^{-1} l_{A}$, respectively (see [12]). 


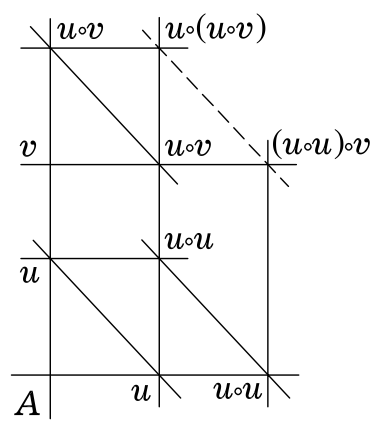

$\left(B_{l}\right)$

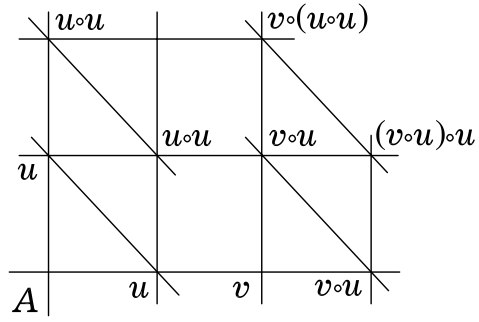

$\left(B_{r}\right)$

FIGURE 5 .

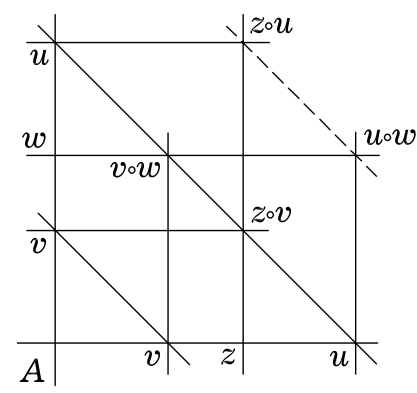

$\left(B_{m}\right)$

Figure 6.

For local loops of Moufang quasigroups, all three conditions (19), (20) and (21) hold simultaneously. Moreover, any two of them imply the third.

The Bol figure $\left(B_{l}\right)$ (see Figure 5 ) is obtained from the Reidemeister figure $(R)$ if one identifies the leaves $u$ and $v$ of the first foliation (see Figure 4). Similarly, the Bol figure $\left(B_{r}\right)$ (see Figure 5 ) is obtained from the figure $(R)$ if one identifies the leaves $v$ and $w$ of the second foliation. The Bol figure $\left(B_{m}\right)$ (see Figure 6 ) is obtained from the figure $(R)$ if one identifies the leaves of the third foliation, i.e., for $z \circ v=v \circ w$, where $z=u / v$. As a result, conditions (19), (20) and (21) are weakened versions of associativity condition (16).

Conditions (19), (20) and (21) show that in local algebras of Bol quasigroups one of the following three conditions:

$$
(\xi, \xi, \eta)=0, \quad(\xi, \eta, \xi)=0, \quad(\xi, \eta, \eta)=0
$$

holds, and the tensors $b_{j k l}^{i}$ satisfy one of the following three relations:

$$
b_{(j k) l}^{i}=0, \quad b_{(j|k| l)}^{i}=0, \quad b_{j(k l)}^{i}=0 .
$$

The local algebras of the Bol quasigroups are called Bol algebras. Bol algebras were studied in detail in [45] (see also [46] and [42]) and 222.

Bol algebras have two independent operations, commutation and association, which are related by a series of algebraic conditions. For Bol quasigroups, the operations in the prolonged algebra $L^{\prime}(Q)=L^{(4)}(Q)$ are expressed in terms of the 
operations of commutation and association. Hence the $G$-structures determined by Bol quasigroups are closed $G$-structures of third order.

On three-webs defined by Bol quasigroups, the closure conditions called the Bol closure conditions $\left(B_{l}\right),\left(B_{r}\right)$ and $\left(B_{m}\right)$ (see Figures 5 and 6 ) hold (see [15, 12], 8], 24). The latter conditions were obtained by Bol as far back as 1936 .

On the manifold $M=Q \times Q$, Bol quasigroups define a structure of a symmetric space. The relation between Bol loops and symmetric spaces was investigated in [45, 22] and in the book [29] (see also the book [36]).

5. Monoassociative quasigroups. A quasigroup, all of whose local loops are monoassociative, i.e., the identity

$$
(u \circ u) \circ u=u \circ(u \circ u)
$$

holds on them, is said to be monoassociative.

It follows that the associators of local algebras of monoassociative quasigroups satisfy the condition

$$
(\xi, \xi, \xi)=0
$$

which is equivalent to the condition

$$
b_{(j k l)}^{i}=0 .
$$

The local algebra $L(Q)$ of such a quasigroup does not define a closed $G$-structure on $Q$. A closed $G$-structure on $Q$ is defined by the prolonged algebra $L^{\prime}(Q)=$ $L^{(4)}(Q)$ (see Section 5) defined in a fourth-order neighborhood of the local loop $l_{A}$. Besides the operation of commutation and association, by means of (15) taken for $s=4$, the algebra $L^{\prime}(Q)$ defines two more independent quaternary operations called the left and right quaternators (see [11). These operations are defined by the fourth-order terms of the Taylor decomposition (12) of the operation of multiplication in $Q$. All other operations defined by the terms of (12) of order higher than four are algebraically expressed by means of the commutators, associators and quaternators of the algebra $L^{\prime}(Q)$. Hence the $G$-structures determined by monoassociative quasigroups are closed $G$-structures of fourth order.

The two operations of quaternation are connected with the operations of commutation and association by a series of relations. These relations were investigated by Shelekhov [49, Mikheev [32] and in [12] (Ch. 7) (see also [9], Section 8).

Three-webs defined by monoassociative quasigroups are called hexagonal, and the hexagonal closure condition (see Figure 7) holds on them (see [15], 12], 8], 24]). Hexagonal three-webs on two-dimensional manifolds were studied by Blaschke and his coworkers as far back as 1927 (see [14]). The history of web geometry started from the study of hexagonal three-webs. It was proven in 14 that on twodimensional three-webs (i.e., in the case $r=1$ ) the hexagonality condition implies web parallelizability. This is not true on multidimensional three-webs, i.e., in the case $r>1$ (see [12]).

The quasigroups and their local algebras which we considered in this section allow us to formulate the following generalized third converse Lie theorem.

Theorem 7. The local Akivis algebras $L(Q)$ associated with Moufang and Bol quasigroups determine these quasigroups in a unique way. The monoassociative quasigroups are determined uniquely only by the prolonged Akivis algebras $L^{\prime}(Q)$.

In fact, multiplication in a Moufang quasigroup is determined by decomposition (12), all terms of which are expressed in terms of the commutators of the algebra 


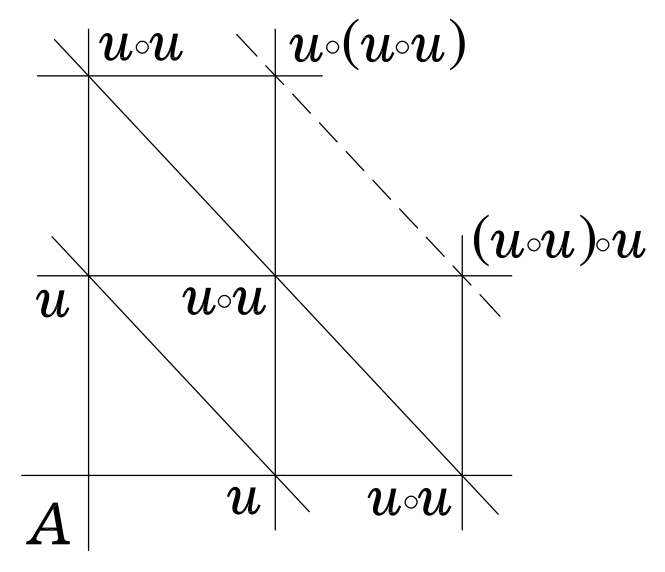

\section{$(H)$}

FIGURE 7.

$L(Q)$, connected by Sagle's identity (18). Multiplication in a Bol quasigroup is determined by the decomposition (12), all terms of which are expressed in terms of the commutators and associators of the algebra $L(Q)$ connected by the identities obtained in 22 and [46. The derivatives in a monoassociative quasigroup are expressed in terms of the commutators, associators and quaternators of its local algebra $L^{\prime}(Q)$ connected by the certain identities (see above for references).

For Moufang quasigroups the generalized third converse Lie theorem was proven by Kuz'min (see [28]), and for Bol loops this theorem was proven by Sabinin and Mikheev [45] (see also [46], pp. 75-77, and [33, section XII.8).

As we noted in Section 4, in general, an Akivis algebra does not completely determine the associated quasigroup.

\section{Shestakov's TheOREM}

Let $B$ be an algebra with a binary multiplication which is not associative. In $B$, we consider the commutators $[x, y]=x \cdot y-y \cdot x$ and the associators $(x, y, z)=$ $(x \cdot y) \cdot z-x \cdot(y \cdot z)$. One can easily check that these operations satisfy relations (10) and (11). Hence the algebra $\operatorname{Ak}(B)$ defined by these two operations is an Akivis algebra. Akivis in [4] posed the following problem:

Problem. Is it true that an arbitrary Akivis algebra can be isomorphically embedded into the algebra $\operatorname{Ak}(B)$ of some noncommutative algebra $B$ ?

If an Akivis algebra admits such a representation, it is called linear. Hofmann and Strambach in [26] formulated a weakened version of Akivis' problem: is a free Akivis algebra linear?

In [50] and [51] (see also [52]), Shestakov gave a positive solution to these two problems. In addition, in the joint papers [53, and [54, the authors considered primitive elements of nonassociative algebras and hyperalgebras introduced by Sabinin and Mikheev in [47] (see also [33], section XII.7 and [41, Ch. 4) and formulated a 
problem similar to Akivis' problem for hyperalgebras. However, there is no complete solution of this problem in [53] and [54]: the authors only outline a way to solve the problem.

In [54, the authors noted that Lie algebras, Mal'cev algebras and Bol algebras are hyperalgebras in the sense of [47] (see also [33, section XII.7).

The prolonged local algebras $L^{(s)}(Q)$ of differentiable quasigroups $Q$ considered in the current paper are also hyperalgebras in the sense of [47].

\section{Differentiable quasigroups on Grassmannians}

We now consider the real projective space $\mathbb{P}^{r+1}$ of dimension $r+1$ and the set of all straight lines in this space. The latter set is the Grassmannian $\mathbb{G}(1, r+1)$. Its dimension is $2 r: \operatorname{dim} \mathbb{G}(1, r+1)=2 r$. The bundles $S(x)$ of straight lines with centers $x \in \mathbb{P}^{r+1}$ are linear submanifolds in $\mathbb{G}(1, r+1)$ of dimension $r: \operatorname{dim} S(x)=r$.

A smooth hypersurface $X \subset \mathbb{P}^{r+1}$ determines a foliation on the Grassmannian $\mathbb{G}(1, r+1)$. The leaves of this foliation are the bundles $S(x)$ of straight lines with centers at points $x \in X$.

We consider three smooth hypersurfaces $X_{\alpha}, \alpha=1,2,3$, in the space $\mathbb{P}^{r+1}$ which are in general position. The set of straight lines in $\mathbb{P}^{r+1}$ intersecting each of the hypersurfaces $X_{\alpha}$ at a point form a domain $M$ on the Grassmannian $\mathbb{G}(1, r+1)$. The hypersurfaces $X_{\alpha}$ define on $M$ three foliations, each of which is formed by the intersections of bundles of straight lines $p$ with centers at points $x_{\alpha} \in X_{\alpha}$ with the manifold $M$ (see Figure 8).

Let $\alpha, \beta, \gamma$ be a permutation of the numbers $1,2,3$. Then for each choice of points $x_{\alpha} \in X_{\alpha}$ and $x_{\beta} \in X_{\beta}$, the straight lines from $M$ determine a point $x_{\gamma} \in X_{\gamma}$. Thus the straight lines from $M$ define a mapping

$$
f_{\gamma}: X_{\alpha} \times X_{\beta} \rightarrow X_{\gamma}
$$

which is a local differentiable quasigroup $Q(G)$.

Using the moving frame method (see 2] and also [12, Section 3.3), one can prove that the commutator and the associator of the quasigroup $Q(G)$ have the following

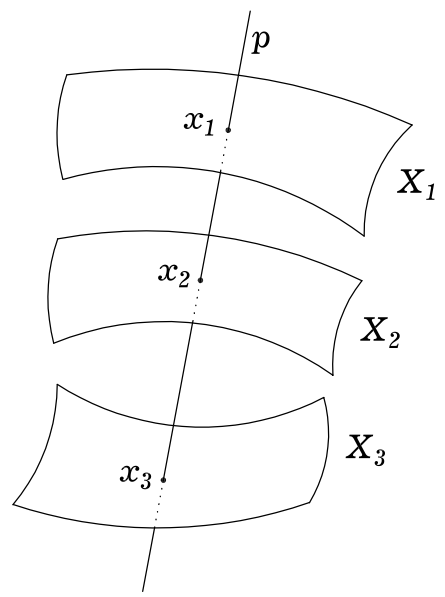

FIGURE 8. 
forms:

$$
[\xi, \eta]=a(\xi) \eta-a(\eta) \xi
$$

and

$$
(\xi, \eta, \zeta)=h_{1}(\eta, \zeta) \xi+h_{2}(\zeta, \xi) \eta+h_{3}(\xi, \eta) \zeta,
$$

where the linear form $a(\xi)$ is defined by the mutual location of the tangent spaces to the hypersurfaces $X_{\alpha}$ at the points $x_{\alpha}$ belonging to the same straight line from $M$, and the symmetric bilinear forms $h_{\alpha}(\xi, \eta)$ are the second fundamental forms of the hypersurfaces $X_{\alpha}$ at points $x_{\alpha} \in X_{\alpha}$.

Definition 8. A quasigroup $Q$ is called Grassmannizable if it is isomorphic to a quasigroup $Q(G)$ defined by a triple of hypersurfaces $X_{\alpha}$ in the space $\mathbb{P}^{r+1}$.

Theorem 9. A quasigroup $Q$ is Grassmannizable if and only if in all its local algebras the operations of commutation and association are expressed by formulas (25) and (26).

Every two-dimensional general position plane $\pi$ of the space $\mathbb{P}^{r+1}$ intersects the hypersurfaces $X_{\alpha}$ along three curves $C_{\alpha}$. The curves $C_{\alpha}$ define in the plane $\pi$ a three-web $W(\pi)$ formed by three two-dimensional bundles of straight lines. The web $W(\pi)$ is dual to a rectilinear three-web $W\left(\pi^{*}\right)$ on the dual plane $\pi^{*}$ (see Figures 9 and 10).

A three-web $W$ carrying the maximal number of two-dimensional subwebs is called transversally geodesic (see [12]). A Grassmannizable three-web possesses the maximal number of two-dimensional subwebs. Hence a Grassmannizable three-web is transversally geodesic.

Consider a few particular cases of Grassmannizable three-webs.

(i) Suppose that a Grassmannizable three-web is hexagonal. As we proved in Section 6 , on hexagonal webs the condition $(\xi, \xi, \xi)=0$ holds. By (26), this condition reduces to the form

$$
h_{1}(\xi, \xi)+h_{2}(\xi, \xi)+h_{3}(\xi, \xi)=0 .
$$

Because a symmetric bilinear form is completely determined by the corresponding quadratic form, the last condition is equivalent to the equation

$$
h_{1}(\xi, \eta)+h_{2}(\xi, \eta)+h_{3}(\xi, \eta)=0
$$

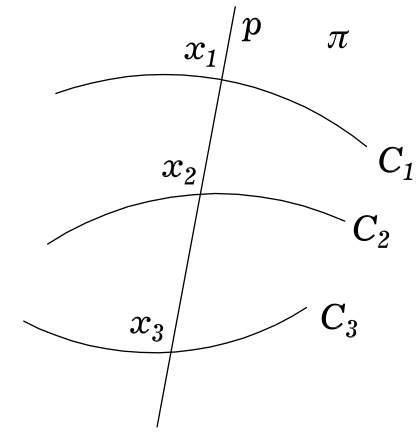

Figure 9.

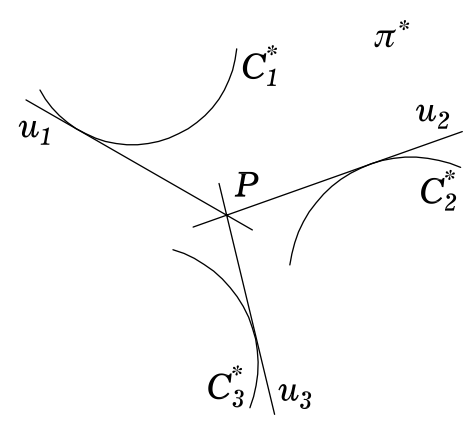

Figure 10. 
Moreover, in this case all two-dimensional three-webs $W(\pi)$ are hexagonal, as well as their dual rectilinear three-webs $W\left(\pi^{*}\right)$. But by the Graf-Sauer theorem (see [15]), such a web is formed by the tangents to a third class curve. Therefore, the triple of curves $C_{\alpha}$, on which the centers of the bundles $S\left(x_{\alpha}\right)$ generating the three-web $W(\pi)$ lie, belongs to one and the same cubic curve. Hence the hypersurfaces $X_{\alpha}$ themselves belong to one and the same cubic hypersurface $U(3)$ of the space $\mathbb{P}^{r+1}$.

Because the converse statement is also true, we proved the following theorem.

Theorem 10. A Grassmannizable quasigroup $Q(G)$ is monoassociative, and the three-web $W(G)$ defined by $Q(G)$ is hexagonal, if and only if the hypersurfaces $X_{\alpha} \subset \mathbb{P}^{r+1}$, generating the quasigroup $Q(G)$ and the threeweb $W(Q)$, belong to one and the same cubic hypersurface $U(3)$.

By Theorem 9, condition (27) is the condition of algebraizability of a triple of hypersurfaces $X_{\alpha} \subset \mathbb{P}^{r+1}, \alpha=1,2,3$. This result is a multidimensional generalization of the Reiss theorem proven in 1837 for a system of curves in the plane (see [38; see also 7], Sections 8.1 and 8.2, where one can find further references and the history of this theorem).

(ii) Suppose that the three-web $W(G)$ is a Bol web and the corresponding quasigroup $Q(G)$ is a Bol quasigroup. Then on this web, one of conditions (22) holds.

Consider, for example, the first of conditions (22) corresponding to the left Bol quasigroup $\left(B_{l}\right)$. The associator of such a quasigroup satisfies the condition $(\xi, \xi, \eta)=0$. Then relation (26) takes the form

$$
\left(h_{1}(\xi, \eta)+h_{2}(\xi, \eta)\right) \xi+h_{3}(\xi, \eta) \eta=0 .
$$

Since the vectors $\xi$ and $\eta$ are linearly independent, it follows that

$$
h_{1}(\xi, \eta)+h_{2}(\xi, \eta)=0, \quad h_{3}(\xi, \eta)=0 .
$$

Thus, the cubic hypersurface $U(3) \subset \mathbb{P}^{r+1}$ generating the three-web $W(G)$ in question decomposes into the hyperquadric $U(2)$ and the hyperplane $X_{3}$.

(iii) Suppose that a Grassmannizable quasigroup $Q(G)$ is a Lie group. Then all three hypersurfaces $X_{\alpha} \subset \mathbb{P}^{r+1}$ generating $Q(G)$ become hyperplanes. The same is true when $Q(G)$ is a Moufang quasigroup.

(iv) Finally, if a Grassmannizable quasigroup $Q(G)$ is an abelian Lie group, then the cubic hypersurface $U(3) \subset \mathbb{P}^{r+1}$ generating $Q(G)($ and $W(G))$ decomposes into three hyperplanes of the same pencil.

The converses of (i)-(iv) are also true. In particular, it follows from this that if the second fundamental forms $h_{\alpha}(\xi, \eta)$ of the hypersurfaces $X_{\alpha} \subset \mathbb{P}^{r+1}$ at their common points with the straight lines of $\mathbb{P}^{r+1}$ satisfy condition (27), then the hypersurfaces $X_{\alpha}$ are parts of one and the same cubic hypersurface $U(3) \subset \mathbb{P}^{r+1}$.

\section{ABOUT THE AUTHORS}

Maks A. Akivis is a professor at Jerusalem College of Technology-Mahon Lev, Israel.

Vladislav V. Goldberg is a distinguished professor of mathematics at New Jersey Institute of Technology. He is a recipient of several research and teaching awards. 
Akivis and Goldberg have authored and coauthored numerous books and papers in differential geometry.

\section{REFERENCES}

1. M. A. Akivis, The canonical expansions of the equations of a local analytic quasigroup (Russian), Dokl. Akad. Nauk SSSR 188 (1969), no. 5, 967-970. English translation: Soviet Math. Dokl. 10 (1969), no. 5, 1200-1203. MR0262413(41:7021) Zbl 205:026013

2. M. A. Akivis, The local differentiable quasigroups and three-webs that are determined by a triple of hypersurfaces (Russian), Sibirsk. Mat. Zh. 14 (1973), no. 3, 467-474. English translation: Siberian Math. J. 14 (1973), no. 3, 319-324. MR0324559|(48:2911) Zbl 267:53005 \& 281:53002.

3. M. A. Akivis, Closed G-structures on a differentiable manifold, Problems in Geometry, 7, 69-79 (Russian), Akad. Nauk SSSR Vsesoyuz. Inst. Nauchn. i Tekhn. Inform., Moscow, 1975. MR0478055 (57:17549) Zbl 549:53032.

4. M. A. Akivis, The local algebras of a multidimensional three-web (Russian), Sibirsk. Mat. Zh. 17 (1976), no. 1, 5-11. English translation: Siberian Math. J. 17 (1976), no. 1, 3-8. MR 0405261 (53:9055) Zbl 337:53018.

5. M. A. Akivis, Geodesic loops and local triple systems in a space with an affine connection, Sibirsk. Mat. Zh. 19 (1978), no. 2, 243-253 (Russian). English translation: Siberian Math. J. 19 (1978), no. 2, 171-178. MR0487840 (58:7438) Zbl 388:53007 \& 409:53008.

6. M. A. Akivis, Bol three-webs and non-Euclidean geometries, Webs and Quasigroups, 16-26, Tver. Gos. Univ., Tver, 1994. MR.1413333 (97f:53024) Zbl 896:53015.

7. M. A. Akivis and V. V. Goldberg, Projective Differential Geometry of Submanifolds, NorthHolland, Amsterdam, 1993, xii+362 pp. MR.1234487(94i:53001) Zbl 865:53013.

8. M. A. Akivis and V. V. Goldberg, Differential geometry of webs, Chapter 1 in Handbook of Differential Geometry, pp. 1-152, Elsevier Science B. V., 2000. MR1736852 (2001f:53036). Zbl 968:53001.

9. M. A. Akivis and V. V. Goldberg, Algebraic aspects of web geometry, Comment. Math. Univ. Carolin. 41 (2000), no. 2, 205-236. MR1780866 (2001i:63026) Zbl 1042:53007.

10. M. A. Akivis and A. M. Shelekhov, Foundations of the Theory of Webs (Russian), Kalinin. Gos. Univ., Kalinin, 1981, 88 pp. MR0639895 (83h:53001) Zbl 475:53016.

11. M. A. Akivis and A. M. Shelekhov, Fourth-order alternators of a local analytic loop and three-webs of multidimensional surfaces (Russian), Izv. Vyssh. Uchebn. Zaved. Mat. 1989, no. 4 (334), 12-16. English translation: Soviet Math. (Iz. VUZ) 33 (1989), no. 4, 13-18. MR:1008142 (90f:53032), Zbl 701:53027.

12. M. A. Akivis and A. M. Shelekhov, Geometry and Algebra of Multidimensional Three-Webs, translated from Russian by V. V. Goldberg, Kluwer Academic Publishers, Dordrecht, 1992, xvii+358 pp. MR.1196908 (93k:53021) Zbl 771:53001.

13. V. D. Belousov, Foundations of the Theory of Quasigroups and Loops, Izdat. "Nauka", Moscow, 1967, 223 pp. (Russian). MR0218483 (36:1569) Zbl 163:01801.

14. W. Blaschke Thomsens Sechseckgewebe. Zueinander diagonale Netze, Math. Z. 28 (1927), 150-157. JFM 54:074404.

15. W. Blaschke and G. Bol, Geometrie der Gewebe. Springer-Verlag, Berlin, 1938, viii+339 pp. Zbl 20:06701.

16. G. Bol, Über Drei-Gewebe im vierdimensionalen Raum. Math. Ann. 110 (1935), 431-463. Zbl 10:22203.

17. R. H. Bruck, A survey of binary systems, Springer-Verlag, Berlin, 1971, 3rd printing, viii + 185 pp. MR0093552 (20:76); Zbl 206:30301.

18. S. S. Chern, Eine Invariantentheorie der Dreigewebe aus $r$-dimensionalen Mannigfaltigkeiten in $\mathbf{R}_{2 r}$, Abh. Math. Sem. Univ. Hamburg 11 (1936), no. 1-2, 333-358. Zbl 13:41802.

19. A. Einstein, Zur Elektrodynamik bewegter Körper, Ann. Phys. (4) 17 (1905), 891-921. JFM 36:092002.

\footnotetext{
${ }^{3}$ In the bibliography we will use the following abbreviations for the review journals: JFM for Jahrbuch für die Fortschritte der Mathematik, MR for Mathematical Reviews, and Zbl for Zentralblatt für Mathematik.
} 
20. L. Euler, Recherches sur une espéce de carrés magiques, in Commentationes Arithmeticae Collectae, vol. II, St. Petersburg Acad. Sci., St. Petersburg, 1849, pp. 302-361; see also reprint made by Kraus Reprint, Nendeln/Liechtenstein, 1969, Vol. I, 1xxxix+584 pp.; Vol. II: ix+651 pp. MR0250831 (40:4063)

21. L. Euler, Recherches sur une nouvelle espèce de quarrés magiques, Verh. Zeeuwsch. Genootsch. Wetensch. Vlissengen Vol. 9 (1782), pp. 85-239; see also Euler, L., Opera Omnia., Series I: Opera mathematica. Vol. VII: algebraicae ad theoriam combinationum et probabilitatum pertinentes (Latin), Teubner, Leipzig and Berlin, 1923, pp. 291-392. JFM 49:0007.

22. V. I. Fedorova, A condition that defines multidimensional Bol three-webs, Sibirsk. Mat. Zh. 19 (1978), no. 4, 922-928 (Russian). English translation: Siberian Math. J. 19 (1978), no. 4, 657-661. MR514146 (58:24036) Zbl 398:53007 \& 409:53007.

23. Ya. Friedman, Physical Applications of Homogenous Balls. With the assistance of T. Scarr, Progress in Mathematical Physics 40, Birkhäuser Boston, Inc., Boston, MA, 2005, xxiv+279 pp. MR2104434 (2005m:32044)

24. V. V. Goldberg, Local differentiable quasigroups and webs, Chapter X in the book Quasigroups and Loops: Theory and Applications, 263-311, Heldermann-Verlag, Berlin, 1990. MR.1125816 (93g:20133) Zbl 737:53015.

25. K. H. Hofmann and K. Strambach, The Akivis algebra of a homogeneous loop, Mathematika 33 (1986), no. 1, 87-95. MR0859501 (88d:17003) Zbl 601:22002.

26. K. H. Hofmann and K. Strambach, Topological and analytic loops, Chapter IX in the book Quasigroups and Loops: Theory and Applications, 205-262, Heldermann-Verlag, Berlin, 1990. MR:0859501 (93g:20133) Zbl 737:53015.

27. M. Kikkawa, On local loops in affine manifolds, J. Sci. Hiroshima Univ. Ser A-I Math. 28 (1964), 199-207. MR0187173 (32:4627) Zbl 141:19603.

28. E. N. Kuz'min, On relation between Mal'cev algebras and analytic Moufang loops, Algebra i Logika 10 (1971), no. 1, 3-22 (Russian). English translation: Algebra and Logic 10 (1971), no. 1, 1-14. MR0297916 (45:6968) Zbl 244:17019 \& 248:17001.

29. O. Loos, Symmetric Spaces, vol. 1 (viii+198 pp.), vol. 2 (viii+193 pp.), Benjamin, New York, 1969. MR0239005 (39:365a,b) Zbl 175:48601.

30. J. Lòhmus, E. Paal and L. Sorgsepp, Nonassociative Algebras in Physics, Hadronic Press, Palm Harbor, FL, xiv+271 pp. MR.1320709 (97d:81095) Zbl 840:17001.

31. A. I. Mal'cev, Analytical loops, Mat. Sb. 36 (1955), 569-576 (Russian). MR0069190(16:997g) Zbl 65:00702.

32. P. O. Mikheev, On a problem of Chern-Akivis-Shelekhov on hexagonal tree-webs, Aequationes Math. 51 (1996), no. 1-2, 1-11. MR1372779 (97a:53019) Zbl 845:53011.

33. P. O. Mikheev and L. V. Sabinin, Quasigroups and differential geometry, Chapter XII in the book Quasigroups and Loops: Theory and Applications, 357-430, Heldermann-Verlag, Berlin, 1990. MR:1125816 (93g:20133) Zbl 737:53015.

34. R. Moufang, Zur Struktur von Alternativkörpern, Math. Ann. 110 (1935), 416-430. Zbl 10:00403.

35. G. P. Nagy, The Campbell-Hausdorff series of local analytic Bruck loops, Abh. Math. Semin. Univ. Hamb. 72 (2002), 79-87. MR1941548 (2003m:20097) Zbl 1015:20050.

36. P. T. Nagy and K. Strambach, Loops in Group Theory and Lie Theory, de Gruyter Expositions in Mathematics, 35, Walter de Gruyter \& Co., Berlin, 2002, xii+361 pp. MR 1899331 (2003d:20110) Zbl 1050:22001.

37. H. O. Pflugfelder, Quasigroups and Loops: Introduction, Heldermann-Verlag, Berlin, 1990, iii+147 pp. MR1125767 (93g:20132) Zbl 715:20043.

38. M. Reiss, Mémoire sur les propriétés générales des courbes algébriques, Correspondance Mathématique et Physique de Quêtelet 9 (1837), 249-308.

39. L. V. Sabinin, The geometry of loops, Mat. Zametki 12 (1972), no. 5, 605-616 (Russian). English translation: Math. Notes 12 (1972), no. 5, 799-805. MR0340461 (49:5216) Zbl 258:20006.

40. L. V. Sabinin, Homogeneous spaces and quasigroups, Izv. Vyssh. Uchebn. Zaved. Mat. 1996, no. 7 (410), 77-84 (Russian). English translation: Soviet Math. (Iz. VUZ) 40 (1996), no. 7, 74-81. MR1430701 (97k:53053) Zbl 874:53037.

41. L. V. Sabinin, Smooth Quasigroups and Loops, Kluwer Academic Publishers, Dordrecht, 1999, xvi+249 pp. MR:1727714(2001e:20069) Zbl 939:20064. 
42. L. V. Sabinin and P. O. Mikheev, Analytic Bol loops, Webs and Quasigroups, 102-109 (Russian), Kalinin. Gos. Univ., Kalinin, 1982. MR0674312 (84c:22007) Zbl 499:20044.

43. L. V. Sabinin and P. O. Mikheev, A symmetric connection in the space of an analytic Moufang loop, Dokl. Akad. Nauk SSSR 262 (1982), no. 4, 807-809 (Russian). English translation: Soviet Math. Dokl. 25 (1982), no. 1, 136-138. MR0644875 (84e:53027) Zbl 495:53048.

44. L. V. Sabinin and P. O. Mikheev, On the geometry of smooth Bol loops, Webs and Quasigroups, 144-154 (Russian), Kalinin. Gos. Univ., Kalinin, 1984. MR0896092 Zbl 568:53009.

45. L. V. Sabinin, and P. O. Mikheev, The differential geometry of Bol loops, Dokl. Akad. Nauk SSSR 281 (1985), 1055-1057 (Russian). English translation: J. Sov. Math. Dokl. 31 (1985), no. 2, 389-391. MR 0787287(86k:53022) Zbl 587:53021.

46. L. V. Sabinin, and P. O. Mikheev, The Theory of Smooth Bol Loops, Dual Russian/English text, Univ. Druzhby Narodov, Moscow, 1985, 81 pp. MR0831661 (87j:22030) Zbl 584:53001.

47. L. V. Sabinin, and P. O. Mikheev, Infinitesimal theory of local analytic loops, Dokl. Akad. Nauk SSSR 297 (1987), no. 4, 801-804 (Russian). English translation: Soviet Math. Dokl. 36 (1988), no. 3, 545-548. MR0924255 (89g:22003) Zbl 659:53018.

48. A. A. Sagle, Mal'cev algebras, Trans. Amer. Math. Soc. 101 (1961), no. 3, 426-458. MR0143791 (26:1343) Zbl 101:02302.

49. A. M. Shelekhov, Classification of multidimensional three-webs according to closure conditions (Russian), Probl. Geom. 21, 109-154, Itogi Nauki i Tekhniki, Akad. Nauk SSSR Vsesoyuz. Inst. Nauchn. i Tekhn. Inform., Moscow, 1989. English translation: Soviet Math. 55 (1991), no. 6, 2140-2168. MR.1027856 (91b:53018) Zbl 705:53014.

50. I. P. Shestakov, Linear representability of Akivis algebras (Russian), Dokl. Akad. Nauk 368 (1999), no. 1, 21-23. English translation: Dokl. Math. 60 (1999), No.2, 164-166. MR1726261 (2001e:17003) Zbl 1034:17500.

51. I. P. Shestakov, Every Akivis algebra is linear, Geom. Dedicata 77 (1999), no. 2, 215-223. MR.1713296 (2000f:17004) Zbl 1043:17002.

52. I. P. Shestakov, Speciality and deformations of algebras, Bahturin, Yu. (ed.), Algebra. Proceedings of the international algebraic conference on the occasion of the 90th birthday of $A$. G. Kurosh, Moscow, Russia, May 25-30, 1998, 345-356, de Gruyter, Berlin, 2000. MR1754680 (2001b:17005) Zbl 1027:17001.

53. I. P. Shestakov and U. U. Umirbaev, Free Akivis algebras, primitive elements, and hyperalgebras, IX Algebra Meeting USP/UNICAMP/UNESP (Portuguese) (São Pedro, 2001), Resenhas 5 (2001), no. 2, 149-158. MR1945299(2003m:17002) Zbl 1034:17002.

54. I. P. Shestakov and U. U. Umirbaev, Free Akivis algebras, primitive elements and hyperalgebras, J. Algebra 250 (2002), no. 2, 533-548. MR1899864(2003c:17005) Zbl 993:17002.

55. A. K. Suschkewitsch, On a generalization of the associative law, Trans. Amer. Math. Soc. 31 (1929), 204-214. JFM 55:008501.

56. A. A. Ungar, Beyond the Einstein Addition Law and its Gyroscopic Thomas Precession. The Theory of Gyrogroups and Gyrovector Spaces, Fundamental Theories of Physics, 117, Kluwer Academic Publishers Group, Dordrecht, 2001, xlii+413 pp. MR.1978122 (2004c:83006) Zbl 972:83002.

57. K. Yamaguti, On algebras of totally geodesic space (Lie triple systems), J. Sci. Hiroshima Univ. Ser A 21 (1957), no. 2, 107-113. MR0100046 (20:6482) Zbl 84:18405.

58. K. Yamaguti, On the Lie triple system and its generalization, J. Sci. Hiroshima Univ. Ser A 21 (1958), 155-160. MR0100047(20:6483) Zbl 84:18404.

Department of Mathematics, Jerusalem College of Technology-Mahon Lev, Havaad Haleumi St., P. O. Box 16031, Jerusalem 91160, Israel

E-mail address: akivis@mail.jct.ac.il

Department of Mathematical Sciences, New Jersey Institute of Technology, University Heights, Newark, New Jersey 07102

E-mail address: vlgold@oak.njit.edu 